\title{
AXILLARY SURGICAL APPROACH IN METASTATIC BREAST CANCER PATIENTS: A SYSTEMATIC REVIEW AND META-ANALYSIS
}

Fabiana C.A.P. Lisboa', Roberta B. Silva², Keitty R. C. de Andrade', Ana Claudia M.G. Figueiredo², Lucimara P. C. Veras 3 , Maurício G. Pereira ${ }^{4}$

'School of Medicine, Universidade de Brasília - Brasília (DF), Brazil.

${ }^{2}$ Faculty of Health Sciences, Universidade de Brasília - Brasília (DF), Brazil.

${ }^{3}$ Foundation of Education and Research in Health Sciences - Brasília (DF), Brazil.

${ }^{4}$ School of Medicine - Brasília (DF), Brazil.

Objective: To evaluate the benefit of an axillary surgical approach on overall survival in patients with metastatic breast cancer. Summary background data: Metastatic breast cancer at diagnosis is considered to be an incurable disease, and systemic treatment is indicated. However, several retrospective studies have shown that local treatment is an independent factor in improving overall survival, but this issue is still controversial. Methods: Systematic review with literature searches in MEDLINE (by PubMed), EMBASE, Clinical Trials, Scopus, Web of Science, SciELO, Lilacs, Google Scholar and Proquest electronic databases, and gray literature without restrictions on date, language or study design. Study selection and data extraction were performed independently by two authors. Data were summarized through meta-analyses using a random effects model and considered hazard ratio (HR) measures and their confidence intervals (95\%CI). Results: After eligibility assessment, we included 16 studies in the systematic review, encompassing a total of 16,692 patients. Twelve studies were used in the quantitative analysis. The studies included in this review seemed to have overall good methodological quality. There was no association between an axillary surgical approach and overall survival (HR=0.82; 95\%CI 0.60-1.13). We identified as a secondary finding that there was a benefit from a breast surgical approach related to overall survival in patients with metastatic breast cancer (HR 0.70; 95\%CI 0.60-0.82). Conclusion: An axillary surgical approach showed no association with overall survival in patients with metastatic breast cancer, but the breast surgical approach seemed to show a benefit in terms of overall survival. The true impact of locoregional therapies on long-term outcomes remains unknown, and randomized clinical trials are needed. 